\title{
On semi-equivalence of generically-finite polynomial mappings
}

\author{
Zbigniew Jelonek ${ }^{1}$
}

Received: 20 September 2014 / Accepted: 28 October 2015 / Published online: 26 November 2015

(C) The Author(s) 2015. This article is published with open access at Springerlink.com

\begin{abstract}
Let $f, g: X \rightarrow Y$ be continuous mappings. We say that $f$ is topologically equivalent to $g$ if there exist homeomorphisms $\Phi: X \rightarrow X$ and $\Psi: Y \rightarrow Y$ such that $\Psi \circ f \circ \Phi=g$. Moreover, we say that $f$ is topologically semi-equivalent to $g$ if there exist open, dense subsets $U, V \subset X$ and homeomorphisms $\Phi: U \rightarrow V$ and $\Psi: Y \rightarrow Y$ such that $\left.\Psi \circ f \circ \Phi\right|_{U}=\left.g\right|_{U}$. Let $X, Y$ be smooth irreducible affine complex varieties. We show that every algebraic family $F: M \times X \ni(m, x) \mapsto F(m, x)=f_{m}(x) \in Y$ of polynomial mappings contains only a finite number of topologically non-equivalent proper mappings and only a finite number of topologically non-semi-equivalent generically-finite mappings. In particular there are only a finite number of classes of topologically non-equivalent proper polynomial mappings $f: \mathbb{C}^{n} \rightarrow \mathbb{C}^{m}$ of a bounded (algebraic) degree. The same is true for a number of classes of topologically non-semi-equivalent generically-finite polynomial mappings $f: \mathbb{C}^{n} \rightarrow \mathbb{C}^{m}$ of a bounded (algebraic) degree.
\end{abstract}

Mathematics Subject Classification 14 D $99 \cdot 14$ R $99 \cdot 51$ M 99

\section{Introduction}

Let $f, g: X \rightarrow Y$ be continuous mappings. We say that $f$ is topologically equivalent to $g$ if there exist homeomorphisms $\Phi: X \rightarrow X$ and $\Psi: Y \rightarrow Y$ such that $\Psi \circ f \circ \Phi=g$. Moreover, we say that $f$ is topologically semi-equivalent to $g$ if there exist open, dense subsets $U, V \subset X$ and homeomorphisms $\Phi: U \rightarrow V$ and $\Psi: Y \rightarrow Y$ such that $\left.\Psi \circ f \circ \Phi\right|_{U}=\left.g\right|_{U}$. In the case $X=\mathbb{C}^{n}$ and $Y=\mathbb{C}$ René Thom stated a Conjecture that there are only finitely many topological types of polynomials $f: X \rightarrow Y$ of bounded degree. This Conjecture was confirmed by Fukuda [2]. Also a more general problem was considered: how many

The author is partially supported by the Narodowe Centrum Nauki Grant, number 2015/17/B/ST1/02637.

Zbigniew Jelonek najelone@cyf-kr.edu.pl

1 Instytut Matematyczny, Polska Akademia Nauk, Śniadeckich 8, 00-656 Warszawa, Poland 
topological types are there in the family $P(n, m, k)$ of polynomial mapping $f: \mathbb{C}^{n} \rightarrow$ $\mathbb{C}^{m}$ of degree bounded by $k$ ? Aoki and Noguchi [1] showed that there are only a finite number of topologically non-equivalent mappings in the family $P(2,2, k)$. Finally Nakai [8] showed that each familiy $P(n, m, k)$, where $n, m, k>3$, contains infinitely many different topological types even if we consider only generically-finite mappings. Hence the General Thom Conjecture is not true even for generically-finite mappings. However, we show in this paper that there are only a finite number of classes of topologically semi-equivalent generically-finite polynomial mappings $f: \mathbb{C}^{n} \rightarrow \mathbb{C}^{m}$ of a bounded (algebraic) degree. As a by product of our considerations we give a simple proof of the following interesting fact: for every $n, m$ and $k$ there are only a finite number of topological types of proper polynomial mappings $f: \mathbb{C}^{n} \rightarrow \mathbb{C}^{m}$ of (algebraic) degree bounded by $k$. Hence we can say that Thom Conjecture is true for proper polynomial mappings. We show also that if $n \leq m$ and $\Omega_{n}\left(d_{1}, \ldots, d_{m}\right)$ denotes the family of all polynomial mappings $F=\left(f_{1}, \ldots, f_{m}\right): \mathbb{C}^{n} \rightarrow \mathbb{C}^{m}$ of a multi-degree bounded by $\left(d_{1}, \ldots, d_{m}\right)$, then any two general member of this family are topologically equivalent.

In fact we prove more: if $X, Y$ are smooth affine irreducible varieties, then every algebraic family $\mathcal{F}$ of polynomial mappings from $X$ to $Y$ contains only a finite number of topologically non-semi-equivalent (non-equivalent) generically-finite (proper) mappings. Moreover, if a family $\mathcal{F}$ is irreducible, then two generic members of $\mathcal{F}$ are in the same equivalence class.

Let us recall here, that a mapping $f: X \rightarrow Y$ is generically finite, if for general $x \in X$ the set $f^{-1}(f(x))$ is finite. Our proof goes as follows. Let $M$ be a smooth affine irreducible variety and let $\mathcal{F}$ be a family of polynomial mappings induced be a regular mapping $F: M \times X \rightarrow Y$, i.e., $\mathcal{F}:=\left\{f_{m}: X \ni x \mapsto F(m, x) \in Y, m \in M\right\}$. Let us recall that if $f: X \rightarrow Z$ is a generically finite polynomial mapping of affine varieties, then the bifurcation set $B(f)$ of $f$ is the set $\left\{z \in Z: z \in \operatorname{Sing}(Z)\right.$ or $\left.\# f^{-1}(z) \neq \mu(f)\right\}$, where $\mu(f)$ is the topological degree of $f$. The set $B(f)$ is always closed in $Z$. We show that there exists a Zariski open, dense subset $U$ of $M$ such that

(1) for every $m \in U$ we have $\mu\left(f_{m}\right)=\mu(\mathcal{F})$, where we treat $f_{m}$ as a mapping $f_{m}: X \rightarrow$ $Z_{m}:=\overline{f_{m}(X)}$

(2) for every $m_{1}, m_{2} \in U$ the pairs $\left(\overline{f_{m_{1}}(X)}, B\left(f_{m_{1}}\right)\right)$ and $\left(\overline{f_{m_{2}}(X)}, B\left(f_{m_{2}}\right)\right)$ are equivalent via a homeomorphism, i.e., there is a homeomorphism $\Psi: Y \rightarrow Y$ such that $\Psi\left(\overline{f_{m_{1}}(X)}\right)=\overline{f_{m_{2}}(X)}$ and $\Psi\left(B\left(f_{m_{1}}\right)\right)=B\left(f_{m_{2}}\right)$.

In particular the group $G=\pi_{1}\left(\overline{f_{m}(X)} \backslash B\left(f_{m}\right)\right)$ does not depend on $m \in U$. Using elementary facts from the theory of topological coverings, we show that the number of topological semi-types (types) of generically-finite (proper) mappings in the family $\mathcal{F}_{\mid U}$ is bounded by the number of subgroups of $G$ of index $\mu(\mathcal{F})$, hence it is finite. Then we conclude the proof by induction. Finally, the case of arbitrary $M$ can be easily reduced to the smooth, irreducible, affine case.

Remark 1.1 In this paper we use the term "polynomial mapping" for every regular mapping $f: X \rightarrow Y$ of affine varieties.

\section{Bifurcation set}

Let $X, Z$ be affine irreducible varieties of the same dimension and assume that $X$ is smooth. Let $f: X \rightarrow Z$ be a dominant polynomial mapping. It is well known that there is a Zariski open non-empty subset $U$ of $Z$ such that for every $x_{1}, x_{2} \in U$ the fibers $f^{-1}\left(x_{1}\right), f^{-1}\left(x_{2}\right)$ 
have the same number $\mu(f)$ of points. We say that $\mu(f)$ is the topological degree of $f$. Recall the following (see $[5,6])$.

Definition 2.1 Let $X, Z$ be as above and let $f: X \rightarrow Z$ be a dominant polynomial mapping. We say that $f$ is finite at a point $z \in Z$ if there exists an open neighborhood $U$ of $z$ such that the mapping $f_{\mid f^{-1}(U)}: f^{-1}(U) \rightarrow U$ is proper.

It is well-known that the set $S_{f}$ of points at which the mapping $f$ is not finite is either empty or it is a hypersurface (see [5,6]). We say that $S_{f}$ is the set of non-properness of $f$.

Definition 2.2 Let $X$ be a smooth affine $n$-dimensional variety and let $Z$ be an affine variety of the same dimension. Let $f: X \rightarrow Z$ be a generically finite dominant polynomial mapping of geometric degree $\mu(f)$. The bifurcation set of $f$ is

$$
B(f)=\left\{z \in Z: z \in \operatorname{Sing}(Z) \text { or } \# f^{-1}(z) \neq \mu(f)\right\} .
$$

Remark 2.3 The same definition makes sense for those continuous mapping $f: X \rightarrow Z$, for which we can define the topological degree $\mu(f)$ and singularities of $Z$. In particular if $Z_{1}, Z_{2}$ are affine algebraic varieties, $f: X \rightarrow Z_{1}$ is a dominant polynomial mapping and $\Phi: Z_{1} \rightarrow Z_{2}$ is a homeomorphism which preserves singularities, then we can define $B(\Phi \circ f)$ as $\Phi(B(f))$. Moreover, the mapping $\Phi \circ f$ behaves topologically as an analytic covering. We will use this facts in the proof of Theorem 3.5.

We have the following theorem (see also [7]).

Theorem 2.4 Let $X, Z$ be affine irreducible complex varieties of the same dimension and suppose $X$ is smooth. Let $f: X \rightarrow Z$ be a polynomial dominant mapping. Then the set $B(f)$ is closed and $B(f)=K_{0} \cup S_{f} \cup \operatorname{Sing}(Z)$.

Proof Let us note that outside the set $S_{f} \cup \operatorname{Sing}(Z)$ the mapping $f$ is a (ramified) analytic covering of degree $\mu(f)$. By Lemma 2.5 below, if $z \notin \operatorname{Sing}(Z)$ we have $\# f^{-1}(z) \leq \mu(f)$. Moreover, since $f$ is an analytic covering outside $S_{f} \cup \operatorname{Sing}(Z)$ we see that for $y \notin S_{f} \cup$ $\operatorname{Sing}(Z)$ the fiber $f^{-1}(z)$ has exactly $\mu(f)$ points counted with multiplicity. Take $X_{0}:=$ $X \backslash f^{-1}\left(\operatorname{Sing}(Z) \cup S_{f}\right)$. If $z \in K_{0}\left(f_{\mid X_{0}}\right)$, the set of critical values of $f_{\mid X_{0}}$, then $\# f^{-1}(z)<$ $\mu(f)$.

Now let $z \in S_{f} \backslash \operatorname{Sing}(Z)$. There are two possibilities:

(a) $\# f^{-1}(z)=\infty$.

(b) $\# f^{-1}(z)<\infty$.

In case (b) we can assume that $f^{-1}(z) \neq \emptyset$. Let $U$ be an affine neighborhood of $z$ disjoint from $\operatorname{Sing}(z)$ over which the mapping $f$ has finite fibers. Let $V=f^{-1}(U)$. By the Zariski Main Theorem in the version given by Grothendieck (see [3]), there exists a normal variety $\bar{V}$ and a finite mapping $\bar{f}: \bar{V} \rightarrow U$ such that

(1) $V \subset \bar{V}$,

(2) $\bar{f}_{\mid V}=f$.

Since $z \in \bar{f}(\bar{V} \backslash V)$, it follows from Lemma 2.5 below that $\# f^{-1}(z)<\mu(f)$. Consequently, if $z \in S_{f}$, we have $\# f^{-1}(z)<\mu(f)$. Finally, we have $B(f)=K_{0}\left(f_{\mid X_{0}}\right) \cup S_{f} \cup$ $\operatorname{Sing}(Z)$. However, the set $K_{0}\left(f_{\mid X_{0}}\right)$ is closed in $Z \backslash\left(S_{f} \cup \operatorname{Sing}(Z)\right)$. Hence $B(f)$ is closed in $Z$.

Lemma 2.5 Let $X, Z$ be affine normal varieties of the same dimension. Let $f: X \rightarrow Z$ be a finite mapping. Then for every $z \in Z$ we have $\# f^{-1}(z) \leq \mu(f)$. 
Proof Let $\# f^{-1}(z)=\left\{x_{1}, \ldots, x_{r}\right\}$. We can choose a function $h \in \mathbb{C}[X]$ which separates all $x_{i}$ (in particular we can take as $h$ the equation of a general hyperplane section). Since $f$ is finite, the minimal equation of $h$ over the field $\mathbb{C}(Z)$ is of the form:

$$
T^{s}+a_{1}(f) T^{s-1}+\cdots+a_{s}(f) \in f^{*} \mathbb{C}[Z][T],
$$

where $s \leq \mu(f)$. If we substitute $f=z$ into this equation we get the desired result.

\section{Main result}

We start with the following:

Lemma 3.1 Let $f: X^{k} \rightarrow Y^{l}$ be a dominant polynomial mapping of affine irreducible varieties. There exists a Zariski open non-empty subset $U \subset Y$ such that for any $y \in U$ we have $\operatorname{Sing}\left(f^{-1}(y)\right)=f^{-1}(y) \cap \operatorname{Sing}(X)$.

Proof We can assume that $Y$ is smooth. Since there exists a mapping $\pi: Y^{l} \rightarrow \mathbb{C}^{l}$ which is generically etale, we can assume that $Y=\mathbb{C}^{l}$. Let us recall that if $Z$ is an algebraic variety, then a point $z \in Z$ is smooth if and only if the local $\operatorname{ring} \mathcal{O}_{z}(Z)$ is regular, or equivalently $\operatorname{dim}_{\mathbb{C}} \mathfrak{m} / \mathfrak{m}^{2}=\operatorname{dim} Z$, where $\mathfrak{m}$ denotes the maximal ideal of $\mathcal{O}_{Z}(Z)$.

Let $y=\left(y_{1}, \ldots, y_{l}\right) \in \mathbb{C}^{l}$ be a sufficiently generic point. Then by Sard's Theorem the fiber $Z=f^{-1}(y)$ is smooth outside $\operatorname{Sin} g(X)$ and $\operatorname{dim} Z=\operatorname{dim} X-l=k-l$. Note that the generic (scheme-theoretic) fiber $F$ of $f$ is reduced. Indeed, this fiber $F=\operatorname{Spec}\left(\mathbb{C}(Y) \otimes_{\mathbb{C}[Y]} \mathbb{C}[X]\right)$ is the spectrum of a localization of $\mathbb{C}[X]$ and so a domain. Since we are in characteristic zero, the reduced $\mathbb{C}(Y)$-algebra $\mathbb{C}(Y) \otimes \mathbb{C}[Y] \mathbb{C}[X]$ is necessarily geometrically reduced (i.e. stays reduced after extending to an algebraic closure of $\mathbb{C}(Y)$ ). Since the property of fibres being geometrically reduced is open on the base, i.e. on $Y$, thus the fibres over an open subset of $Y$ will be reduced. Consequently, there is a Zariski open, non-empty subset $U \subset Y$ such that for $y \in U$ the fiber $f^{-1}(y)$ is reduced. Hence we can assume that $Z$ is reduced. It is enough to show that every point $z \in Z \cap \operatorname{Sing}(X)$ is singular on $Z$.

Assume that $z \in Z \cap \operatorname{Sing}(X)$ is smooth on $Z$. Let $f: X \rightarrow \mathbb{C}^{l}$ be given as $f=$ $\left(f_{1}, \ldots, f_{l}\right)$, where $f_{i} \in \mathbb{C}[X]$. Then $\mathcal{O}_{z}(Z)=\mathcal{O}_{z}(X) /\left(f_{1}-y_{1}, \ldots, f_{l}-y_{l}\right)$. In particular if $\mathfrak{m}^{\prime}$ denotes the maximal ideal of $\mathcal{O}_{z}(Z)$ and $\mathfrak{m}$ denotes the maximal ideal of $\mathcal{O}_{z}(X)$ then $\mathfrak{m}^{\prime}=\mathfrak{m} /\left(f_{1}-y_{1}, \ldots, f_{l}-y_{l}\right)$. Let $\alpha_{i}$ denote the class of the polynomial $f_{i}-y_{i}$ in $\mathfrak{m} / \mathfrak{m}^{2}$. Let us note that

$$
\mathfrak{m}^{\prime} / \mathfrak{m}^{\prime 2}=\mathfrak{m} /\left(\mathfrak{m}^{2}+\left(\alpha_{1}, \ldots, \alpha_{l}\right)\right) .
$$

Since the point $z$ is smooth on $Z$ we have $\operatorname{dim}_{\mathbb{C}} \mathfrak{m}^{\prime} / \mathfrak{m}^{\prime 2}=\operatorname{dim} Z=\operatorname{dim} X-l$. Take a basis $\beta_{1}, \ldots, \beta_{k-l}$ of the space $\mathfrak{m}^{\prime} / \mathfrak{m}^{\prime 2}$ and let $\overline{\beta_{i}} \in \mathfrak{m} / \mathfrak{m}^{2}$ correspond to $\beta_{i}$ under the correspondence (1). Note that the vectors $\overline{\beta_{1}}, \ldots, \overline{\beta_{k-l}}, \alpha_{1}, \ldots, \alpha_{l}$ generate the space $\mathfrak{m} / \mathfrak{m}^{2}$. This means that $\operatorname{dim}_{\mathbb{C}} \mathfrak{m} / \mathfrak{m}^{2} \leq k-l+l=k=\operatorname{dim} X$. Hence the point $z$ is smooth on $X$, a contradiction.

We have:

Lemma 3.2 Let $X, Y$ be smooth complex irreducible algebraic varieties and $f: X \rightarrow Y$ a regular dominant mapping. Let $N \subset W \subset X$ be closed subvarieties of $X$. Then there exists a non-empty Zariski open subset $U \subset Y$ such that for every $y_{1}, y_{2} \in U$ the triples $\left(f^{-1}\left(y_{1}\right), W \cap f^{-1}\left(y_{1}\right), N \cap f^{-1}\left(y_{1}\right)\right)$ and $\left(f^{-1}\left(y_{2}\right), W \cap f^{-1}\left(y_{2}\right), N \cap f^{-1}\left(y_{2}\right)\right)$ are homeomorphic. 
Proof Let $X_{1}$ be an algebraic completion of $X$ and let $\bar{Y}$ be a smooth algebraic completion of $Y$. Take $X_{1}^{\prime}:=\overline{\operatorname{graph}(f)} \subset X_{1} \times \bar{Y}$ and let $X_{2}$ be a desingularization of $X_{1}^{\prime}$.

We can assume that $X \subset X_{2}$. We have an induced mapping $\bar{f}: X_{2} \rightarrow \bar{Y}$ such that $\bar{f}_{\mid X}=f$. Let $Z=X_{2} \backslash X$. Denote by $\bar{N}, \bar{W}$ the closures of $N$ and $W$ in $X_{2}$. Let $\mathcal{R}=$ $\{\bar{N} \cap Z, \bar{W} \cap Z, \bar{N}, \bar{W}, Z\}$, a collection of algebraic subvarieties of $X_{2}$. There is a Whitney stratification $\mathcal{S}$ of $X_{2}$ which is compatible with $\mathcal{R}$.

For any smooth strata $S_{i} \in \mathcal{S}$ let $B_{i}$ be the set of critical values of the mapping $\bar{f}_{\mid S_{i}}$ and denote $B=\overline{\cup B_{i}}$. Take $X_{3}=X_{2} \backslash \bar{f}^{-1}(B)$. The restriction of the stratification $\mathcal{S}$ to $X_{3}$ gives a Whitney stratification which is compatible with the family $\mathcal{R}^{\prime}:=\mathcal{R} \cap X_{3}$. We have a proper mapping $f^{\prime}:=\bar{f}_{\mid X_{3}}: X_{3} \rightarrow \bar{Y} \backslash B$ which is a submersion on each stratum. By the Thom first isotopy theorem there is a trivialization of $f^{\prime}$ which preserves the strata. It is an easy observation that this trivialization gives a trivialization of the mapping $f: X \backslash f^{-1}(B) \rightarrow Y \backslash B:=U$. In particular the fibers $f^{-1}\left(y_{1}\right)$ and $f^{-1}\left(y_{2}\right)$ are homeomorphic via a stratum preserving homeomorphism. This means that the triples $\left(f^{-1}\left(y_{1}\right), W \cap f^{-1}\left(y_{1}\right), N \cap f^{-1}\left(y_{1}\right)\right)$ and $\left(f^{-1}\left(y_{2}\right), W \cap f^{-1}\left(y_{2}\right), N \cap f^{-1}\left(y_{2}\right)\right)$ are homeomorphic.

We also need the following:

Definition 3.3 Let $X, Y$ be smooth affine varieties. By a family of regular mappings $\mathcal{F}_{M}(X, Y, F):=\mathcal{F}$ we mean a regular mapping $F: M \times X \rightarrow Y$, where $M$ is an algebraic variety. The members of a family $\mathcal{F}$ are the mappings $f_{m}: X \ni x \rightarrow F(m, x) \in Y$. Let

$$
G: M \times X \ni(m, x) \mapsto(m, F(m, x)) \in Z=\overline{G(M \times X)} \subset M \times Y .
$$

If $G$ is generically finite, then by the topological degree $\mu(\mathcal{F})$ we mean the number $\mu(G)$. Otherwise we put $\mu(\mathcal{F})=0$.

Later we will sometimes identify the mapping $f_{m}$ with the mapping $G(m, \cdot)=\left(m, f_{m}\right)$ : $X \rightarrow m \times Y$. The following lemma is important:

Lemma 3.4 Let $X, Y$ be smooth affine complex varieties. Let $M$ be a smooth affine irreducible variety and let $\mathcal{F}$ be the family induced by a mapping $F: M \times X \rightarrow Y$, i.e., $\mathcal{F}=\left\{f_{m}: X \ni x \mapsto F(m, x) \in Y, m \in M\right\}$. Assume that $\mu(\mathcal{F})>0$. Take $Z=\overline{G(M \times X)}$ and put $Z_{m}=(m \times Y) \cap Z$.

Then

(1) There is an open non-empty subset $U_{1} \subset M$ such that for every $m \in U_{1}$ we have $\mu\left(f_{m}\right)=\mu(\mathcal{F})$

(2) There is a non-empty open subset $U_{2} \subset U_{1}$ such that for every $m \in U_{2}$ we have $\overline{f_{m}(X)}=Z_{m}:=(m \times Y) \cap Z$ and $B\left(f_{m}\right)=B(G)_{m}:=(m \times Y) \cap B(G)$;

(3) There is a non-empty open subset $U_{3} \subset U_{2}$ such that for every $m_{1}, m_{2} \in U_{3}$ the pairs $\left(\overline{f_{m_{1}}(X)}, B\left(f_{m_{1}}\right)\right)$ and $\left(\overline{f_{m_{2}}(X)}, B\left(f_{m_{2}}\right)\right)$ are equivalent by means of a homeomorphism, i.e., there is a homeomorphism $\Psi: Y \rightarrow Y$ such that $\Psi\left(\overline{f_{m_{1}}(X)}\right)=\overline{f_{m_{2}}(X)}$ and $\Psi\left(B\left(f_{m_{1}}\right)\right)=B\left(f_{m_{2}}\right)$.

Proof (1) Take $G: M \times X \ni(m, x) \mapsto(m, F(m, x)) \in Z$. The mapping $G: M \times X \ni$ $(m, x) \mapsto(m, F(m, x)) \in Z$ has a constant number of points in the fibers outside the bifurcation set $B(G) \subset Z$. Take $U=Z \backslash B(G)$. By Theorem 2.4 the set $U$ is open. Let $\pi: Z \ni(m, y) \mapsto m \in M$ be the projection. We show that the constructible set $\pi(U)$ is dense in $M$. Indeed, assume that $\overline{\pi(U)}=N$ is a proper subset of $M$. Since $U$ is dense in $Z$, we have $\pi(Z) \subset N$, i.e., $Z \subset N \times Y$. This is a contradiction. In particular the set $\pi(U)$ is dense in $M$ and it contains a Zariski open, non-empty subset $U_{1} \subset M$. Of course $\mu\left(f_{m}\right)=\mu(\mathcal{F})$ for $m \in U_{1}$. 
(2) Consider the projection $\pi: Z \ni(m, y) \mapsto m \in M$. As we know from (1), the mapping $\pi$ is dominant. By a well known result, after shrinking $U_{1}$ we can assume that every fiber $Z_{m}$ of $\pi\left(m \in U_{2} \subset U_{1}\right)$ is of pure dimension $d=\operatorname{dim} Z-\operatorname{dim} M=\operatorname{dim} X$. However, $Z_{m}=\overline{f_{m}(X)} \cup B(G)_{m}$. Generically the dimension of $B(G)_{m}$ is less than $d$. Hence if we possibly shrink $U_{2}$, we get $Z_{m}=\overline{f_{m}(X)}$ for $m \in U_{2}$. Moreover, by Lemma 3.1 (after shrinking $U_{2}$ if necessary), we can assume that $\operatorname{Sing}\left(Z_{m}\right)=\operatorname{Sing}(Z)_{m}:=$ $(m \times Y) \cap \operatorname{Sing}(Z)$. Now it is easy to see that $B\left(f_{m}\right)=B(G)_{m}$.

(3) We have $\overline{f_{m}(X)}=Z_{m}$ and $B\left(f_{m}\right)=B(G)_{m}$ for $m \in U_{2}$. Now apply Lemma 3.2 with $X=U_{2} \times Y, W=\left(U_{2} \times Y\right) \cap Z, N=\left(U_{2} \times Y\right) \cap B(G)$ and $f: U_{1} \times Y \ni(m, y) \mapsto$ $m \in U_{1}$.

Now we are ready to prove our main result:

Theorem 3.5 Let $X, Y$ be smooth affine irreducible varieties. Every algebraic family $\mathcal{F}$ of polynomial mappings from $X$ to $Y$ contains only a finite number of topologically non-semiequivalent (non-equivalent) generically-finite (proper) mappings.

Proof The proof is by induction on $\operatorname{dim} M$. We can assume that $M$ is affine, irreducible and smooth. Indeed, $M$ can be covered by a finite number of affine subsets $M_{i}$, and we can consider the families $\mathcal{F}_{\mid M_{i}}$ separately. For the same reason we can assume that $M$ is irreducible. Finally $\operatorname{dim} M \backslash \operatorname{Reg}(M)<\operatorname{dim} M$ and we can use induction to reduce the general case to the smooth one.

Assume that $M$ is smooth and affine. If $\mu(\mathcal{F})=0$, then $\mathcal{F}$ does not contain any genericallyfinite mapping. Hence we can assume that $\mu(\mathcal{F})=k>0$. By Lemma 3.4 there is a non-empty open subset $U \subset M$ such that for every $m_{1}, m_{2} \in U$ we have

(1) $\mu\left(f_{m_{1}}\right)=\mu\left(f_{m_{2}}\right)=k$,

(2) The pairs $\left(\overline{f_{m_{1}}(X)}, B\left(f_{m_{1}}\right)\right)$ and $\left(\overline{f_{m_{2}}(X)}, B\left(f_{m_{2}}\right)\right)$ are equivalent by means of a homeomorphism, i.e., there is a homeomorphism $\Psi: Y \rightarrow Y$ such that $\Psi\left(\overline{f_{m_{1}}(X)}\right)=\overline{f_{m_{2}}(X)}$ and $\Psi\left(B\left(f_{m_{1}}\right)\right)=B\left(f_{m_{2}}\right)$.

Fix a pair $Q=\overline{f_{m_{0}}(X)}, B=B\left(f_{m_{0}}\right)$ for some $m_{0} \in U_{3}$. For $m \in U_{3}$ the mapping $f_{m}: X \rightarrow Y$ is topologically equivalent to the continuous mapping $f_{m}^{\prime}=\Psi_{m} \circ f_{m}$ with $\overline{f_{m}^{\prime}(X)}=Q$ and $B\left(f_{m}^{\prime}\right)=B$ (Lemma 3.4). Every mapping $f_{m}^{\prime}$ induces a topological covering $f_{m}^{\prime}: X \backslash f_{m}^{\prime-1}(B)=P_{f_{m}^{\prime}} \rightarrow R=Q \backslash B$. Take a point $a \in R$ and let $a_{f_{m}^{\prime}} \in f_{m}^{\prime-1}(a)$. We have an induced homomorphism

$$
f_{*}: \pi_{1}\left(P_{f_{m}^{\prime}}, a_{f_{m}^{\prime}}\right) \rightarrow \pi_{1}(R, a) .
$$

Denote $H_{f}=f_{*}\left(\pi_{1}\left(P_{f}, a_{f}\right)\right)$ and $G=\pi_{1}(R, a)$. Hence $\left[G: H_{f}\right]=k$. It is well known that the fundamental group of a smooth algebraic variety is finitely generated. In particular the group $G:=\pi_{1}(Q \backslash B, a)$ is finitely generated. Let us recall the following result of $\mathrm{M}$. Hall (see [4]):

Lemma 3.6 Let $G$ be a finitely generated group and let $k$ be a natural number. Then there are only a finite number of subgroups $H \subset G$ such that $[G: H]=k$.

By Lemma 3.6 there are only a finite number of subgroups $H_{1}, \ldots, H_{r} \subset G$ with index $k$. Choose generically-finite (proper) mappings $f_{i}=f_{m_{i}}^{\prime}=\Psi_{i} \circ f_{m_{i}}: X \rightarrow Y$ such that $H_{f_{i}}=H_{i}$ (of course only if such a mapping $f_{i}$ does exist). We show that every genericallyfinite (proper) mapping $f_{m}^{\prime}(m \in U)$ is semi-equivalent (equivalent) to one of mappings $f_{i}$. 
Indeed, let $H_{f_{m}^{\prime}}=H_{f_{i}}$ (here $f_{m}^{\prime}=\Psi_{m} \circ f_{m}$ ). We show that $f_{m}^{\prime}:=f$ is equivalent to $f_{i}$. Let us consider two coverings $f:\left(P_{f}, a_{f}\right) \rightarrow(R, a)$ and $f_{i}:\left(P_{f_{i}}, a_{f_{i}}\right) \rightarrow(R, a)$. Since $f_{*}\left(\pi_{1}\left(P_{f}, a_{f}\right)\right)=f_{i_{*}}\left(\pi_{1}\left(P_{f_{i}}, a_{f_{i}}\right)\right)$ we can lift the covering $f$ to a homeomorphism $\phi: P_{f} \rightarrow P_{f_{i}}$ such that following diagram commutes:

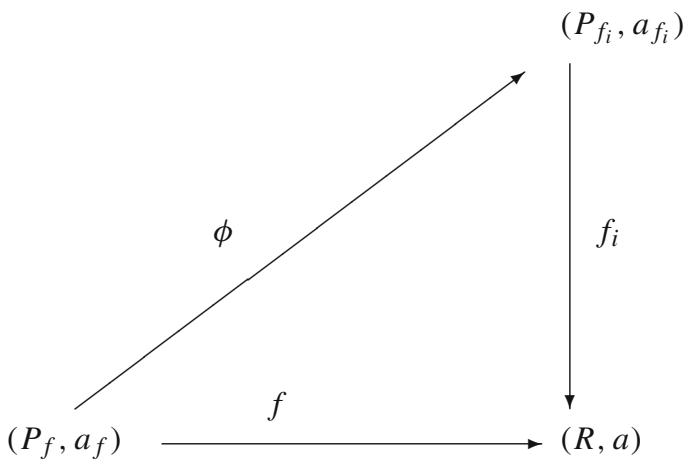

Hence for generically-finite mappings we have

$$
\left.\left(\Psi_{i}\right)^{-1} \circ \Psi_{m} \circ f_{m} \circ \phi^{-1}\right|_{U}=\left.f_{m_{i}}\right|_{U},
$$

where $V=X \backslash f_{m}^{-1}\left(B\left(f_{m}\right)\right)$ and $U=X \backslash f_{m_{i}}^{-1}\left(B\left(f_{m_{i}}\right)\right)$. Hence $f_{m}$ is semi-equivalent to $f_{m_{i}}$.

In the case of proper mappings we show additionally that the mapping $\phi$ can be extended to a continuous mapping $\Phi$ on the whole of $X$. Indeed, take a point $x \in f^{-1}(B)$ and let $y=f(x)$. The set $f_{i}^{-1}(y)=\left\{b_{1}, \ldots, b_{s}\right\}$ is finite. Take small open disjoint neighborhoods $W_{i}(r)$ of $b_{i}$, such that $W_{i}(r)$ shrinks to $b_{i}$ as $r$ tends to 0 . We can choose an open neighborhood $V(r)$ of $y$ so small that $f_{i}^{-1}(V(r)) \subset \bigcup_{j=1}^{s} W_{i}(r)$. Now take a small connected neighborhood $P_{x}(r)$ of $x$ such that $f\left(P_{x}(r)\right) \subset V(r)$. The set $P_{x}(r) \backslash f^{-1}(B)$ is still connected and it is transformed by $\phi$ into one particular set $W_{i_{0}}(r)$. We take $\Phi(x)=b_{i_{0}}$. It is easy to see that the mapping $\Phi$ so defined is a continuous extension of $\phi$. In fact $\phi\left(P_{x}(r) \backslash f^{-1}(B)\right)$ shrinks to $b_{i_{0}}$ if $r$ goes to 0 . Moreover, we still have $f=f_{i} \circ \Phi$.

In a similar way the mapping $\Lambda$ determined by $\phi^{-1}$ is continuous. It is easy to see that $\Lambda \circ \Phi=\Phi \circ \Lambda=$ identity, hence $\Phi$ is a homeomorphism. Consequently, the mapping $f_{i} \circ \Phi=\Psi_{i} \circ f_{m_{i}} \circ \Phi$ is equal to $f=\Psi_{m} \circ f_{m}$. Finally, we get

$$
\left(\Psi_{i}\right)^{-1} \circ \Psi_{m} \circ f_{m} \circ \Phi^{-1}=f_{m_{i}} .
$$

This means that the family $\mathcal{F}_{\mid U}$ contains only a finite number of topologically non-semiequivalent (non-equivalent) generically-finite (proper) mappings. In fact, the number of topological semi-types (types) of generically-finite (proper) mappings in $\mathcal{F}_{\mid U}$ is bounded by the number of subgroups of $G$ of index $\mu(\mathcal{F})$.

Let $T=M \backslash U$. Hence $\operatorname{dim} T<\operatorname{dim} M$. By the induction the family $\mathcal{F}_{\mid T}$ also contains only a finite number of topologically non-semi-equivalent (non-equivalent) generically-finite (proper) mappings. Consequently so does $\mathcal{F}$.

Corollary 3.7 There is only a finite number of topologically non-semi-equivalent (nonequivalent) generically-finite (proper) polynomial mappings $f: \mathbb{C}^{n} \rightarrow \mathbb{C}^{m}$ of a bounded algebraic degree. 


\section{Families of proper mappings}

In this section we slightly extend our previous result in the case of irreducible families of proper (or generically-finite) mappings. First we prove a following lemma:

Lemma 4.1 Let $Y=\mathbb{R}^{n}$ and let $Z \subset Y$ be a linear subspace of $Y$. Fix $\epsilon>0$ and take $\eta<\epsilon$. Let $B(0, \eta)$ be a ball of radius $\eta$. Let $\gamma: I \ni t \mapsto \gamma(t) \in B(0, \eta) \cap Z$ be a smooth path. Then there exists a continuous family of diffeomorphisms $\Phi_{t}: Y \rightarrow Y, t \in[0,1]$ such that

(1) $\Phi_{t}(\gamma(t))=\gamma(0)$ and $\Phi_{t}(z)=z$ for $\|z\| \geq \epsilon$.

(2) $\Phi_{0}=$ identity.

(3) $\Phi_{t}(Z)=Z$.

Proof Let $v_{t}=\gamma(0)-\gamma(t) \in T \mathbb{R}^{n}$. We construct a family of diffeomorphisms $\Phi_{t}$, which are interpolation between translation $x \rightarrow x+v_{t}$ and identity.

Let $\sigma: Y \rightarrow[0,1]$ be a differentiable function such that $\sigma=1$ on $B(0, \eta)$ and $\sigma=0$ outside $B(0, \epsilon)$. Define a vector field $V(x)=\sigma(x) v_{t}$. Integrating this vector field we get desired diffeomeorphisms $\Phi_{t}$, for any $t$.

Corollary 4.2 Let $Y$ be a smooth manifold and $Z$ be a smooth submanifold. For every point $a \in Z$ and every open neighborhood $V_{a}$ of the point a, there is an open connected neighborhood $U_{a}$ of the point a, such that:

(a) $\overline{U_{a}} \subset V_{a}$,

(b) if $\gamma: I \ni t \mapsto \gamma(t) \in U_{a} \cap Z$ is a smooth path, then there is a continuous family of diffeomorphism $\psi_{t}: Y \rightarrow Y, t \in[0,1]$ such that

(1) $\psi_{t}(\gamma(t))=\gamma(0)$

(2) $\psi_{t}(x)=x$ for $x \notin V_{a}$ and $\psi_{0}=$ identity,

(3) $\psi_{t}(Z)=Z$.

Now we are in a position to prove:

Theorem 4.3 Let $X, Y$ be smooth affine irreducible varieties. Let $\mathcal{F}: M \times X \rightarrow Y$ be an algebraic family of proper polynomial mappings from $X$ to $Y$. Assume that $M$ is an irreducible variety. Then there exists a Zariski open dense subset $U \subset M$ such that for every $m, m^{\prime} \in U$ mappings $f_{m}$ and $f_{m^{\prime}}$ are topologically equivalent.

Proof We follow the proof of Theorem 3.5 and we use here the same notation. By Lemma 3.4 there is a non-empty open subset $U \subset M$ such that for every $m_{1}, m_{2} \in U$ we have

(1) $\mu\left(f_{m_{1}}\right)=\mu\left(f_{m_{2}}\right)=k$,

(2) The pairs $\left(\overline{f_{m_{1}}(X)}, B\left(f_{m_{1}}\right)\right)$ and $\left(\overline{f_{m_{2}}(X)}, B\left(f_{m_{2}}\right)\right)$ are equivalent by means of a homeomorphism, i.e., there is a homeomorphism $\Psi: Y \rightarrow Y$ such that $\Psi\left(\overline{f_{m_{1}}(X)}\right)=\overline{f_{m_{2}}(X)}$ and $\Psi\left(B\left(f_{m_{1}}\right)\right)=B\left(f_{m_{2}}\right)$.

Fix a pair $\left(Q=\overline{f_{m_{0}}(X)}, B=B\left(f_{m_{0}}\right)\right)$ for some $m_{0} \in U$. For $m \in U$ the mappings $f_{m}$ and $f_{m_{0}}$ can be connected by a continuous path $f_{t}, f_{0}=f_{m_{0}}, f_{1}=f_{m}$. Moreover we have also a continuous family of homeomorphisms $\Psi_{t}: Y \rightarrow Y$ such that $\Psi_{t}\left(\overline{f_{t}(X)}\right)=\overline{f_{0}(X)}$ and $\Psi\left(B\left(f_{t}\right)\right)=B\left(f_{0}\right)$. It is enough to prove that mappings $F_{t}=\Psi_{t} \circ f_{t}$ are locally (in the sense of parameter $t$ ) equivalent.

(1) First step of the proof. Let $C_{t} \subset X$ denotes the preimage by $F_{t}$ of the set $B$ (in fact $C_{t}=f_{t}^{-1}\left(B\left(f_{t}\right)\right)$ and put $X_{t}=X \backslash C_{t}$. Put $Q^{\prime}:=Q \backslash B$. Assume that for all mappings $F_{t}$ there is a point $a \in\left(X \backslash \bigcup_{t \in I} C_{t}\right)$ such that for all $t \in I$ we have $F_{t}(a)=b$. 
We have an induced homomorphism $G_{t *}: \pi_{1}\left(X_{t}, a\right) \rightarrow \pi_{1}\left(Q^{\prime}, b\right)$. We show that the subgroup $F_{t *}\left(\pi_{1}\left(X_{t}, a\right)\right) \subset \pi_{1}\left(Q^{\prime}, b\right)$ does not depend on $t$.

Indeed let $\gamma_{1}, \ldots, \gamma_{s}$ be generators of the group $\pi_{1}\left(X_{t_{0}}, a\right)$. Let $U_{i}$ be an open relatively compact neighborhoods of $\gamma_{i}$ such that $\overline{U_{i}} \cap C_{t_{0}}=\emptyset$. For sufficiently small number $\epsilon>0$ and $t \in\left(t_{0}-\epsilon, t_{0}+\epsilon\right)$ we have $\overline{U_{i}} \cap C_{t}=\emptyset$. Let $t \in\left(t_{0}-\epsilon, t_{0}+\epsilon\right)$. Note that the loop $F_{t}\left(\gamma_{i}\right)$ is homotopic with the loop $F_{t_{0}}\left(\gamma_{i}\right)$. In particular the group $F_{t_{0 *}}\left(\pi_{1}\left(X_{t_{0}}, a\right)\right)$ is contained in the group $F_{t *}\left(\pi_{1}\left(X_{t}, a\right)\right)$. Since they have the same (finite!) index in $\pi_{1}\left(Y^{\prime}, b\right)$ they are equal. This means that the subgroup $G_{t *}\left(\pi_{1}\left(X_{t}, a\right)\right) \subset \pi_{1}\left(Y^{\prime}, b\right)$ is locally constant, hence it is constant.

Let us consider two coverings $F_{t}:\left(X_{t}, a\right) \rightarrow\left(Q^{\prime}, b\right)$ and $F_{0}:\left(X_{0}, a\right) \rightarrow\left(Q^{\prime}, b\right)$. Since $F_{t *} \pi_{1}\left(X_{t}, a\right)=F_{0 *} \pi_{1}\left(X_{0}, a\right)$ we can lift the covering $F_{t}$ to a homeomorphism $\phi_{t}: X_{t} \rightarrow$ $X_{0}$. As before we can extend the homeomorphism $\phi_{t}$ to the homeomorphism $\Phi_{t}: X \rightarrow X$, such that $F_{0} \circ \Phi_{t}=F_{t}$.

(2) The general case. Now we can prove Theorem 4.3. Since in general there is no a point $a \in\left(X \backslash \bigcup_{t \in I} C_{t}\right)$ such that for all $t \in I$ we have $F_{t}(a)=b$, we have to modify our construction.

First we prove that for every $t_{0} \in I$ there exists $\epsilon>0$ and a family of homeomorphisms $\Phi_{t}: X \rightarrow X, t \in\left(t_{0}-\epsilon, t_{0}+\epsilon\right)$ such that $F_{t}=F_{t_{0}} \circ \Phi_{t}$ for $t \in\left(t_{0}-\epsilon, t_{0}+\epsilon\right)$. Take a point $a \in X_{t_{0}}$ and choose $\epsilon>0$ so small that $a \in X_{t}$ for $t \in\left(t_{0}-\epsilon, t_{0}+\epsilon\right)$. Put $\gamma(t) \ni t \mapsto F_{t}(a) \in Y^{\prime}$. We can take $\epsilon$ so small that the hypothesis of Corollary 4.2 is satisfied. Applying Corollary 4.2 with $Y^{\prime}=Y \backslash B$ and $Z=Q \backslash B$ we have a continuous family of diffeomeorphisms $\psi_{t}: Y \rightarrow Y$ which preserves $Q$ and $B, t \in\left(t_{0}-\epsilon, t_{0}+\epsilon\right)$ such that $\psi_{t}\left(F_{t}(a)\right)=F_{0}(a)$. Take $G_{t}=\psi_{t} \circ F_{t}$. Arguing as in the first part of our proof all $G_{t}$ are topologically equivalent for $t \in\left(t_{0}-\epsilon, t_{0}+\epsilon\right)$. Hence also all $F_{t}$ are topologically equivalent for $t \in\left(t_{0}-\epsilon, t_{0}+\epsilon\right)$. Since $F_{t}$ are locally topologically equivalent, they are topologically equivalent for every $t \in I$.

Corollary 4.4 Let $n \leq m$ and let $\Omega_{n}\left(d_{1}, \ldots, d_{m}\right)$ denotes the family of all polynomial mappings $F=\left(f_{1}, \ldots, f_{m}\right): \mathbb{C}^{n} \rightarrow \mathbb{C}^{m}$ of a multi-degree bounded by $\left(d_{1}, \ldots, d_{m}\right)$. Then any two general members of this family are topologically equivalent.

Proof Indeed, it is enough to note that a generic mapping $f \in \Omega_{n}\left(d_{1}, \ldots, d_{m}\right)$ is proper.

Using the same method we can prove:

Theorem 4.5 Let $X, Y$ be smooth affine irreducible varieties. Let $\mathcal{F}: M \times X \rightarrow Y$ be an algebraic family of generically-finite polynomial mappings from $X$ to $Y$. Assume that $M$ is an irreducible variety. Then there exists a Zariski open dense subset $U \subset M$ such that for every $m, m^{\prime} \in U$ the mappings $f_{m}$ and $f_{m^{\prime}}$ are topologically semi-equivalent.

Open Access This article is distributed under the terms of the Creative Commons Attribution 4.0 International License (http://creativecommons.org/licenses/by/4.0/), which permits unrestricted use, distribution, and reproduction in any medium, provided you give appropriate credit to the original author(s) and the source, provide a link to the Creative Commons license, and indicate if changes were made.

\section{References}

1. Aoki, K., Noguchi, H.: On topological types of polynomial map germs of plane to plane. Mem. Sch. Sci. Eng. Waseda Univ. 44, 133-156 (1980) 
2. Fukuda, T.: Types topologiques des polynomes. Ins. Hautes Études Publ. Math. 46, 87-106 (1976)

3. Grothendieck, A.: EGA IV, Etude locale de schemas et de morphismes de schemes. Publ. Math. IHES 28, 5-255 (1966)

4. Hall, M.: A topology for free groups and related topics. Ann. Math. 52, 127-139 (1950)

5. Jelonek, Z.: The set of points at which a polynomial map is not proper. Ann. Polon. Math. 58, 259-266 (1993)

6. Jelonek, Z.: Testing sets for properness of polynomial mappings. Math. Ann. 315, 1-35 (1999)

7. Jelonek, Z., Kurdyka, K.: Quantitative generalized Bertini-Sard theorem for smooth affine varieties. Discret. Comput. Geom. 34, 659-678 (2005)

8. Nakai, I.: On topological types of polynomial mappings. Topology 23, 45-66 (1984)

9. Thom, R.: La stabilite topologique des applications polynomiales. Enseign. Math. 8, 24-33 (1962) 